\title{
La educación de habilidades sociales desde la Extensión Universitaria. Propuesta de acciones
}

\section{A educação de habilidades sociais a partir da Extensão Universitária. Proposta de ações}

\section{The education of social skills from academic Extension. Proposed actions}

\author{
Mayra Ordaz Hernández ${ }^{1}$
}

\begin{abstract}
RESUMEN
El proceso de extensión universitaria es para la Educación Superior el camino que da cumplimiento a su encomienda social, promoviendo la elevación del nivel cultural a partir de la participación del hombre como agente activo de su propio desarrollo. La presente investigación tuvo como objetivo diseñar acciones para la educación del Trabajo en Equipo desde el Programa de Extensión Universitaria del Departamento de Estudios Socioculturales. Se efectuó el estudio teórico del objeto teniendo en cuenta el tratamiento de la temática en Cuba y en el mundo. Se analizaron documentos, se aplicaron entrevistas a directivos y profesores y se desarrolló un grupo de discusión con estudiantes, a partir de los cuales se pudo constatar que no había presencia del tema en los programas y proyectos rectores de los procesos extensionistas de aprendizaje. Los implicados en la confección de dichos documentos alegaron que era necesaria la enseñanza de habilidades sociales para convivir de manera adecuada en la universidad, pero desconocían las técnicas e instrumentos para su entrenamiento. Por su parte, los estudiantes expresaron que querían aprenderlas, las consideraban necesarias, pero fundamentaron que sus profesores no lo hacían de manera sistemática y organizada. Tomando en cuenta esta realidad, se diseñaron acciones extensionistas dentro del Programa del Departamento de Estudios Socioculturales con el objetivo de
\end{abstract} Noviembre.

1 Universidad de Pinar del Río. Pinar del Río, Cuba. Calle Martí, nº 270. Esq. 27 de 
educar habilidades sociales para el desarrollo de la responsabilidad social de sus estudiantes.

Palabras-clave: habilidades sociales; extensión universitaria; Estudios Socioculturales.

\title{
RESUMO
}

O processo de extensão universitária é para a Educação Superior o caminho em direção à execução de sua função social, promovendo a elevação do nível cultural a partir da participação do homem como agente ativo de seu próprio desenvolvimento. A presente pesquisa teve como objetivo projetar ações para a educação do Trabalho em Equipe a partir do Programa de Extensão Universitária do Departamento de Estudos Socioculturais.. Foi feito um estudo teórico do objeto tendo em mente o tratamento dado ao assunto em Cuba e no mundo. Foram analisados documentos, aplicadas entrevistas a diretores e professores e se desenvolveu um grupo de discussão com estudantes, a partir nos quais se pode constatar-se que não havia a presença deste assunto nos programas e projetos que regem os processos extensionistas de aprendizagem. Os envolvidos na elaboração dos documentos alegaram que é necessário o ensino de habilidades sociais para conviver de modo adequado na universidade, mas ao mesmo tempo eles não conhecem as técnicas e instrumentos para esse treinamento. Por outro lado, os estudantes declararam que querem aprender essas habilidades, pois as consideram necessárias, mas destacararam que seus professores não as ensinam de maneira sistemática e organizada. Levando em conta essa realidade, foram projetadas várias ações extensionistas dentro do Programa do Departamento de Estudos Socioculturais, com o objetivo de trabalhar as habilidades sociais para o desenvolvimento da responsabilidade social dos estudantes.

Palavras-chave: habilidades sociais; extensão universitária; Estudos Socioculturais.

\begin{abstract}
The process of university extension is the road for Superior Education to execute its social function, promoting the elevation of the cultural level from men's participation as an active agent of its own development. The goal of this research was to design actions to promote teamwork in the University Extension Program of the Sociocultural Studies Department. A theoretical study of the object was made, having in mind the treatment given to the subject in Cuba and abroad. Several documents were analyzed, some interviews were applied to directors and professors, and a student discussion group was created, from which it was concluded that there was no presence of this
\end{abstract}


subject in the fundamental programs and projects of the extended learning processes. Those implied in the elaboration of the documents stated that the proper teaching of social skills is needed in the university but, at the same time, they didn't know the techniques and instruments for this training. On the other hand, the students have declared they wanted to learn these skills, they considered to need them, but their professors didn't teach these skills in a systematic and organized way. Therefore, several extended actions were planned within the framework of the Program of the Sociocultural Studies Department, aiming at teaching the social skills for the development of the social responsibility of the students.

Keywords: social skills; university extension; Sociocultural Studies.

\section{Introducción}

Toda relación social es un proceso interactivo y constructor tanto de la identidad personal como colectiva. Esta construcción se lleva a cabo a través del lenguaje, influenciado, como nosotros, por el contexto, la sociedad y el momento histórico en el que se vive. Las relaciones sociales constituyen procesos básicamente psicosociales que tienen la finalidad de unir y trazar relaciones entre los hombres lo suficientemente estables como para que se puedan formar colectividades, tanto en lo que es común, como en las diferencias.

Basta analizar la historia de la humanidad para percibir que gran parte de los conflictos y desacuerdos entre los hombres provienen de la incapacidad para crear y mantener relaciones adecuadas con los demás.

Así, entre la educación y el desarrollo social existen complejos nexos, que condicionan la necesidad actual de que los sistemas educativos den respuesta a las exigencias sociales del siglo XXI. Para lograrlo, se necesita el surgimiento de nuevas alternativas con vistas a perfeccionar la eficiencia de las políticas, sistemas y estrategias educacionales.

Delors ([1996?]), en el Informe de la Comisión Internacional para la Educación del Siglo XXI, a la UNESCO (Organización de las Naciones Unidas para la Educación, la Ciencia y la Cultura), afirma que la educación relacional es un complemento indispensable en el desarrollo cognitivo y una herramienta fundamental de prevención, ya que muchos problemas tienen su origen en el ámbito emocional, por lo que la educación del siglo XXI descansa en cuatro ejes básicos que denomina los cuatro pilares de la educación:

- Aprender a conocer y aprender a aprender para aprovechar las posibilidades de una educación permanente; 
- Aprender a hacer para capacitar a la persona para transferir lo aprendido a situaciones de la vida;

- Aprender a ser, para obrar con autonomía, juicio y responsabilidad personal;

- Aprender a convivir, a trabajar en proyectos comunes y a gestionar la convivencia.

Este último eje, afín directamente con la educación relacional, es un complemento indispensable en el desarrollo cognitivo y una herramienta fundamental de prevención, ya que muchos problemas tienen su origen en el ámbito relacional: a partir de la convivencia nos definimos individual y socialmente.

Para lograr la armonía social, tan necesaria en la cotidianidad humana, es ineludible desarrollar un grupo de habilidades a través de las cuales se generen emociones agradables, se controlen las desagradables, se expresen adecuadamente criterios desfavorables al contexto respetando juicios ajenos, se trabaje en equipo, así como se resuelvan eficazmente problemas profesionales y personales, entre otras muchas destrezas.

Según Goleman (1995, p. 141)

[...] las habilidades de la persona maduran. Estas son las capacidades que contribuyen a la eficacia en el trato con los demás; aquí los déficits conducen a la ineptitud en el mundo social o a los desastres interpersonales repetidos. En efecto, es precisamente la ausencia de estas habilidades lo que puede hacer que incluso los más brillantes intelectualmente fracasen en sus relaciones, apareciendo como arrogantes, desagradables o insensibles. Estas habilidades sociales le permiten a uno dar forma a un encuentro, movilizar e inspirar a otros, prosperar en las relaciones íntimas, persuadir e influir, tranquilizar a los demás.

La educación, en cualquier nivel de enseñanza, sin el aprendizaje de habilidades sociales que fortalezcan las dimensiones intra e interpersonales, no prepara integralmente para la vida, teniendo en cuenta que no es posible una convivencia social adecuada sin que la preceda un aprendizaje sistemático y consciente de aquellas prácticas que la hacen posible. Además, se ha declarado de manera constante, que la educación superior aspira a formar personas socialmente responsables, donde la enseñanza de habilidades sociales cobra vida propia pues, sin éstas, no es posible lograr el comprometimiento de sus estudiantes con el mundo en que viven.

El Trabajo en Equipo 
[...] es la sinergia laboral enfocada hacia la consecución de objetivos colectivos. Las personas dotadas de esta habilidad: alientan cualidades grupales como el respeto, la disponibilidad y la cooperación, estimulan la participación y el entusiasmo, consolidan la identidad grupal y el compromiso, cuidan al grupo y su reputación, y comparten los méritos, cultivan y mantienen amplias redes informales, crean relaciones mutuamente provechosas, buscan y alientan las oportunidades de colaboración, están dispuestas a sacrificarse en aras del objetivo superior de la organización [...]". (GOLEMAN, 1998, p. 283).

El Trabajo en Equipo es una habilidad social que expresa la capacidad del ser humano de trabajar en cooperación con los demás, tan necesaria, como multidisciplinares son las investigaciones científicas en la actualidad. Ya nadie busca verdades solo, es un proceso que se hace en equipo, y poder concebir proyectos de trabajo de manera asertiva, respetando el criterio de los demás y exponiendo el propio, aun cuando no sea del consenso, es una habilidad social necesaria para vivir.

¿Qué es lo que hace que un equipo funcione mucho mejor que el más capacitado de sus integrantes? Esta pregunta resulta clave, porque la actuación de los buenos equipos eleva el "cociente intelectual de grupo" - la suma total de los mejores talentos de sus integrantes - contribuyendo a que den lo mejor de sí mismos. Cuando los equipos funcionan bien los resultados no son tanto aditivos como multiplicativos, ya que los mejores talentos de una persona contribuyen a catalizar los de los demás y terminan produciendo resultados que trascienden con mucho el logro aislado de cualquiera de sus integrantes. Y la explicación de esta faceta de la actuación del grupo reside en la relación entre sus miembros, en la química existente entre ellos (GOLEMAN, 1998, p. 281-282).

En consonancia con este hecho, cabría pensar que la formación de esta habilidad constituya objetivo imprescindible de las instituciones educativas a nivel de cualquier sociedad, desde las primeras edades, hasta la formación profesional de las universidades.

Todo proyecto educativo que busque verdaderamente mejorar la calidad de la enseñanza ha de poner especial interés en la formación cabal de 
las personas, lo que incluye un especial énfasis en el desarrollo de sus habilidades sociales las que, a futuro, serán fundamentales para establecer las redes sociales y de apoyo que requiere la Sociedad de la Información y el espíritu emprendedor. Los hábitos y habilidades sociales que se hagan cargo de lo escolar, lo afectivo, lo vocacional, lo recreativo y lo familiar deben ser tema de primer nivel en toda planificación escolar, hasta la educación superior (DÍAZ; ALUM; ORDAZ, 2013, p. 32-33).

No basta el desarrollo de conocimientos y habilidades técnicas que preparen al hombre para el desempeño laboral vinculado estricta y concretamente al objeto de su profesión, es necesario propiciar una disposición permanente para el análisis y perfeccionamiento del comportamiento comunicativo individual, también asumir una concepción "del otro" que implique respeto, consideración, transparencia, tolerancia y disfrute por las relaciones humanas y a la vez el desarrollo de determinadas cualidades de la personalidad que favorezcan la eficiencia de las relaciones humanas en el contexto laboral.

\section{Una investigación en la Educación Superior para el desarrollo de habilidades sociales}

La Universidad tiene la necesidad de "[...] formar a personas para ser profesionales altamente cualificados para su desempeño laboral y el ejercicio de su ciudadanía de forma responsable, brindando a los estudiantes la posibilidad de desarrollar plenamente sus propias capacidades con sentido de la responsabilidad social" (UNESCO, 1998, p. 7) para ello "[...] la educación superior debe no sólo proporcionar competencias sólidas para el mundo de hoy y de mañana, sino contribuir a la formación de una ciudadanía dotada de principios éticos, comprometidos con la construcción de la paz, la defensa de los derechos humanos y los valores de la democracia." (UNESCO, 2009, p. 9).

En las investigaciones científico-educativas, que a la postre son las encargadas de constituir los pilares sobre los que descansa la práctica pedagógica, no deben pasar por alto aquellos acercamientos que constituyan demostraciones de la ineludible necesidad de colocar al ser humano en el centro mismo de los nuevos descubrimientos educativos.

Los avances alcanzados por la sociedad obligan a repensar los procesos de formación para lograr que los estudiantes universitarios estén en capacidad 
de preservar la cultura desde la docencia, desarrollarla desde la investigación y promoverla desde la dimensión extensionista.

La docencia, la investigación y la extensión universitaria son procesos sustantivos de la universidad y están estrechamente relacionados entre sí. Su integración asegura el cumplimiento de la misión de la Educación Superior en la época actual.

La formación de profesionales en el contexto actual está permeada por los conflictos, crisis y tendencias de la sociedad a nivel mundial, resultado de las relaciones y los acontecimientos sociales que abarcan el mundo contemporáneo.

La universidad actual debe formar profesionales con sólidos principios éticos, y con gran responsabilidad humana y social, que aúnen esfuerzos en lograr un desarrollo sostenible, sin perjuicios al medio ambiente, y que transforme las comunidades en beneficio de todos.

Es por ello que las universidades tienen un papel protagónico en sus respectivas sociedades, en el desarrollo de la conciencia, de la ciencia y de la innovación tecnológica para producir el cambio cualitativo necesario y deseado.

El complejo escenario que es la sociedad actual está marcado por la relación de fenómenos naturales, sociales, culturales, políticos y económicos a escala global. Esta realidad exige la alineación de profesionales con una sólida formación integral, que les permita convertir las comunidades en escenarios indispensables para la promoción cultural y la preservación de lo mejor de nuestras tradiciones.

Si tenemos en cuenta que la universidad en cualquier lugar del mundo es una institución cultural que tiene, como componente del cuerpo social, una marcada responsabilidad con la sociedad que se concreta en actuar por el mejoramiento económico, político, social y cultural como sistema de vida del pueblo, no puede conformarse con el desarrollo de sus procesos de docencia e investigación, sino que también requiere desarrollar el proceso de extensión para dar cumplimiento a su encomienda social, promoviendo la elevación del nivel cultural a partir de la participación del hombre como agente activo de su propio desarrollo.

De esta manera, el nivel de satisfacción del encargo social está directamente relacionado con la madurez y significación que como institución cultural alcance la universidad en su interrelación con la sociedad, en lo que la extensión es un factor clave, pues garantiza un alto grado de solidez a este vínculo. Se considera la extensión universitaria como: "Sistema de interacciones de la Universidad y la Sociedad, mediante la actividad y la comunicación, que se realizan dentro y fuera del centro de educación superior, con el propósito de promover la cultura en la comunidad universitaria y extrauniversitaria, para contribuir a su desarrollo cultural" (GONZÁLEZ; GONZÁLEZ, 1999, p. 28-29). 
El trabajo sociocultural universitario se desarrolla en cuatro niveles básicos de participación, en cada uno de los cuales se expresa el cumplimiento de la función extensionista de la universidad y en los que se manifiestan tanto las propiedades de eficacia, efectividad y eficiencia del proceso extensionista (dimensión administrativa) como las de pertinencia, trascendencia, adecuación e impacto propias de esta labor (dimensión tecnológica).

Estos niveles son: Universidad, Facultad, Departamento y Año Académico (Proyectos Educativos).

Es el Programa de Extensión Universitaria del departamento eslabón imprescindible para la conformación y promoción de las acciones extensionistas que establecerán el verdadero nexo universidad-sociedad.

A partir de todo lo expuesto anteriormente se decidió atender en la presente investigación el proceso extensionista de la Facultad de Ciencias Sociales y Humanísticas de la Universidad de Pinar del Río, a través del análisis del Programa de Extensión Universitaria del Departamento de Estudios Socioculturales, ya que en los proyectos educativos de los colectivos de año no se ponen en práctica acciones orientadas a un trabajo en equipo provechoso desde el punto de vista personal, social y profesional, de esta forma los alumnos no se preparan de manera consciente para crear relaciones mutuamente provechosas, para estimular la participación y el entusiasmo, para trabajar en equipo de manera asertiva ni para negociar de forma adecuada los problemas profesionales y personales.

La metodología cuantitativa está presente en el estudio. No obstante se prestigió esencialmente el paradigma cualitativo con sus técnicas, propio de las ciencias sociales.

También se utilizó la Investigación Acción Participación, que es una herramienta intelectual de transformación social, a través del Grupo de Discusión.

Y los métodos teóricos: Dialéctico, Histórico-lógico, con los procedimientos Análisis Síntesis, Inducción Deducción; y empíricos: Análisis de documentos, Entrevista y Grupo focal o de discusión.

Se analizaron los documentos:

$\checkmark$ Modelo de Formación Profesional de la carrera de Estudios Socioculturales (ESC);

$\checkmark$ Programa Nacional de Extensión Universitaria;

$\checkmark$ Programa de Extensión Universitaria de la Universidad de Pinar del Río (UPR);

$\checkmark$ Programa de Extensión Universitaria de la Facultad de Ciencias Sociales y Humanísticas (FCSH);

$\checkmark$ Programa de Extensión Universitaria del Departamento ESC;

$\checkmark$ Estrategia educativa de la carrera de ESC;

$\checkmark$ Proyectos Educativos de los años académicos; 
$\checkmark$ Diagnóstico de estudiantes en años lectivos.

$\checkmark$ Se entrevistaron los sujetos (muestra no probabilística e intencional):

$\checkmark$ Decano de la FCSH;

$\checkmark$ Vicedecano de Extensión Universitaria;

$\checkmark$ Coordinador de Extensión Universitaria del Departamento de ESC;

$\checkmark$ Jefe del departamento de ESC;

$\checkmark$ Profesores.

Los grupos focales se desarrollaron a partir de una muestra aleatoria probabilística estratificada, desde donde resultó escogido el $20 \%$ de los estudiantes de los tres primeros años de la carrera.

A partir de la instrumentación de los procederes científicos y procesamiento de la información lograda se obtuvo que en el Departamento de Estudios Socioculturales no existen acciones encaminadas a la educación del Trabajo en Equipo de sus estudiantes desde la Extensión Universitaria, de lo que resulta que los Proyectos Educativos de los Colectivos de Año tampoco diseñan tareas orientadas a un trabajo en equipo provechoso desde el punto de vista personal, social y profesional, de esta forma los alumnos no se preparan de manera consciente para crear relaciones mutuamente provechosas, para estimular la participación y el entusiasmo, para trabajar en equipo de manera asertiva, para negociar de forma adecuada los problemas profesionales y personales, para respetar las diferencias, compartir la responsabilidad por las acciones grupales y trabajar por el bien común.

Ante esta situación se formuló el siguiente diseño metodológico:

Problema científico: ¿Cómo contribuir a la educación del Trabajo en Equipo desde la extensión universitaria, en estudiantes de la Licenciatura en Estudios Socioculturales de la Universidad de Pinar del Río?

Objeto de investigación: Proceso de educación del Trabajo en Equipo desde la extensión universitaria.

Objetivo: Diseñar acciones para la educación del Trabajo en Equipo desde el Programa de Extensión Universitaria del Departamento que desarrolle la responsabilidad social universitaria en estudiantes de la Licenciatura en Estudios Socioculturales.

\section{La educación de habilidades sociales en la Extensión Universitaria. Acciones}

La gestión del proceso extensionista en la educación superior es el Trabajo $\underline{\text { Sociocultural Universitario, cuyo contenido es la promoción sociocultural. }}$ 
La extensión universitaria es una manifestación de la relación dialéctica entre la Universidad y la Sociedad, se da en el vínculo, pero no en todo vínculo, sino aquel cuyo fin es la promoción de la cultura; es decir, es aquel en que se establece la relación, entre la cultura y la elevación del nivel cultural de la sociedad en general a través de la función extensionista (PROGRAMA NACIONAL DE EXTENSIÓN UNIVERSITARIA, 2004, p. 7).

La promoción sociocultural cobra vida en la justa medida en que le da sentido a la existencia de la extensión universitaria por el mero hecho de que ésta forma parte integral de la misión formativa de las instituciones de educación superior, por lo que los programas extensionistas deben concebirse, diseñarse y llevarse a cabo en diálogo constante con la comunidad y mediante la plena inmersión de la universidad en la problemática de su sociedad.

De esta forma el carácter comunicacional de la extensión debe tener plena vigencia tanto hacia la propia comunidad universitaria como hacia la sociedad.

Se ha dicho que el imperativo de autenticidad que debe mover a la universidad es el emprendimiento a crear el bien, obligando a diseñar sus programas formativos de manera que correspondan con lo que cabe esperar de una academia de nivel superior.

De esta manera se ha concluido que la extensión es la mejor expresión de una integración creativa Universidad-Sociedad y su vínculo más idóneo, existiendo la posibilidad de hacer de esta función el eje de la acción universitaria y el hilo conductor de la inmersión social de la Universidad, con lo cual adquieren sus programas una extraordinaria relevancia en el quehacer de las instituciones de educación superior.

Es criterio generalizado por parte de los entendidos en la materia que, si la extensión es la función universitaria más próxima a la realidad social, por principio tiene que ejercerse interdisciplinariamente, desde luego que esta es la única manera de acercarse a la realidad, que por naturaleza es interdisciplinaria.

La Universidad para el siglo XXI no es una 'estación de servicios múltiples' ni una 'Cruz Roja académica', dispuesta a atender todas las emergencias. El imperativo de autenticidad [...] que debe mover a la Universidad a emprender sólo aquello que puede hacer bien, obliga a diseñar los programas extensionistas de manera que correspondan con lo que cabe esperar de una academia de nivel superior." (TÜNNERMANN, 1993, p. 25). 
Uno de los fundamentos prácticos en los que se apoya la teoría de que la extensión brinda la estrategia a seguir en la contribución universitaria al desarrollo sociocultural es la consideración de que los modos de actuación profesional son el baluarte sobre el que se deben estructurar las formas concretas de realización de esta contribución.

A partir de estas ideas se diseñan los modos de actuación profesional de cada especialidad y si no se tienen en cuenta estas prácticas, difícilmente el egresado cumpla con la responsabilidad que les corresponde ante la sociedad, como especialista en su área del saber.

El Modelo de Formación Profesional de la Licenciatura en Estudios Socioculturales (ESC) traza los modos de actuación propios de la profesión en la implicación de una sensibilidad especial por la cultura y se caracterizan por una actitud de compromiso con el desarrollo social, de gestión, promoción y trasformación sociocultural facilitadora de la participación activa y el protagonismo de la sociedad en el enriquecimiento espiritual y cultural que la misma necesita en correspondencia con el proyecto social vigente.

La educación de esta actitud de responsabilidad con la sociedad, a través de las acciones que diseña el Programa de Extensión Universitaria del Departamento de ESC, es el objeto de estudio del presente trabajo.

Para ello se escogió el Trabajo en Equipo por ser la habilidad que permite a las personas mantener la afinidad grupal enfocada hacia la consecución de objetivos colectivos, negociando de forma adecuada los problemas profesionales y personales, respetando las diferencias, compartiendo la responsabilidad por las acciones grupales y trabajando por el bien común.

Tal vez no haya lugar en el que se haga evidente con tanta claridad la naturaleza esencial de la coordinación social como en el quehacer científico actual, en donde la puesta a punto de los conocimientos requiere la colaboración eficaz y armónica de todos los implicados (GOLEMAN, 1998, p. 277).

Si no se aprende a trabajar en cooperación con los demás, jamás se contribuirá al progreso social, siendo el perfeccionamiento de la sociedad el avance de todos, entonces todos han de contribuir de manera conjunta a lograrlo.

Lo dicho así pudiera parecer argumento convincente, y lo es, aunque no suficiente: los espacios educativos conscientes, organizados, planificados y sistemáticos (procesos pedagógicos y didácticos) han de educar esta habilidad. La universidad ha de encargarse de enseñar cómo desarrollarla, cómo aprender las operaciones 
necesarias para apropiarse de ella. La institución puede y debe diseñar acciones al interior de sus programas de extensión que conviertan los espacios de la comunidad universitaria en escenarios para aprender y entrenar el Trabajo en Equipo.

Trabajar para el logro de la integralidad universitaria es ocuparse de formar profesionales responsables con sus saberes ante la sociedad, es demostrarles a ellos, en la práctica, la utilidad personal y social de poner sus conocimientos a disposición de la sociedad para el progreso colectivo, es educar la responsabilidad social universitaria desarrollando actitudes de respeto por los demás y compromiso con el bien común. Para ello la educación y entrenamiento de las habilidades sociales y, particularmente, el Trabajo en Equipo, es indispensable para aprender a sacrificarse en aras del objetivo superior de la organización, crear relaciones mutuamente provechosas, estimular la participación y el entusiasmo, consolidar la identidad grupal y el compromiso, compartir los méritos, entre otras habilidades propias de una personalidad equilibrada y creativa.

Toda acción extensionista que comprenda tareas a desarrollar por los estudiantes debe ser dialogada con ellos desde este espacio, desde aquí se debe proponer, sugerir, convenir el día a día universitario donde los estudiantes sean los protagonistas, porque no hay nada más desmotivante que ejecutar acciones que otros hayan diseñado sin consultar los motivos y necesidades propias.

De esta manera se cumple con la dimensión tecnológica de la gestión del proceso extensionista que se estructura a partir de la promoción sociocultural y ésta es un proceso social de práctica, que integra factores psicológicos, sociales, económicos, políticos, culturales; siempre influidos por intereses y valores de su ecosistema, que contribuye a conformarlo y es a su vez conformado por él.

Lo que significa que

[...] los métodos y procedimientos utilizados por esta son los mismos del trabajo social, básicamente apoyados en el estudio de la realidad, de sus problemas, necesidades, recursos y conflictos, la programación de actividades y la acción conducida de manera racional, articulando la tríada "querer-saber-poder" donde se materializa la fusión de lo afectivo, lo conceptual y lo operativo, como sustento de la participación en el ámbito grupal (PROGRAMA NACIONAL DE EXTENSIÓN UNIVERSITARIA, 2004, p. 40).

Para que los estudiantes logren convertirse en promotores culturales desde las diferentes etapas de estudio en que se vinculan con la práctica social, es necesario desarrollar en ellos conocimientos y habilidades básicas que les 
permitan, en su vínculo social, promover la cultura y los avances científico-técnicos de su profesión.

La promoción de la cultura por la vía extracurricular tiene el propósito de complementar el desarrollo cultural integral de los estudiantes. Las actividades extracurriculares promueven la afición y el interés por la cultura, el arte y la literatura; la cultura física, el deporte, la ciencia y el empleo culto del tiempo libre. Estos son espacios donde el estudiante puede aprender con mayor holgura las habilidades sociales, por cuanto son plazas que el estudiante escoge de manera particular, de acuerdo a sus características personales y donde los modos de aprenderlas son muy flexibles. Aquí el trabajo en equipo se hace muy necesario pues los ajustes entre jugadores, especialistas de áreas científicas e integrantes de grupos musicales, son los que deciden el éxito de la tarea que se emprenda.

Difundir los resultados más relevantes de la educación superior, el quehacer de la vida universitaria y social y las actividades extensionistas en la universidad y en la comunidad, para contribuir a la información de la población universitaria y la de su entorno, constituye una de las funciones esenciales de la extensión universitaria.

\section{Propuesta de acciones extensionistas para la educación de habilidades sociales (Trabajo en equipo)}

1. Diagnosticar a estudiantes para el diseño de las veladas político-culturales en conmemoración de fechas históricas;

2. Confeccionar grupos de estudiantes por años académicos para el diseño de las veladas político-culturales en conmemoración de fechas históricas;

3. Crear subgrupos de estudiantes por años académicos que promocionen dentro y fuera de la universidad las acciones que se realizan en los proyectos sociales extra e intrauniversitario de sus respectivas brigadas;

4. Diagnosticar habilidades sociales (TRABAJO EN EQUIPO) en estudiantes por años académicos;

5. Diseñar cursos optativos y facultativos para la educación del Trabajo en Equipo con vistas a elevar la cultura general integral de los estudiantes en los diferentes años de la carrera;

6. Implementar cursos optativos y facultativos en función de las necesidades determinadas en la acción anterior; 
7. Diagnosticar necesidades e intereses de la comunidad universitaria con respecto a los días que para ellos sean de importancia;

8. Crear grupos de estudiantes que se encarguen de organizar y celebrar los días de festividad que se señalen en el diagnóstico;

9. Diagnosticar en los estudiantes de toda la carrera aptitudes para las distintas manifestaciones artísticas: danza, canto, teatro, pintura, cine, artesanía, literatura (poesía, novela, ensayo, cuento y otros);

10. Desarrollar cursos teóricos y prácticos sobre las distintas manifestaciones artísticas;

11. Desarrollar, por parte de los grupos de estudiantes con afinidades artísticas específicas, investigaciones científicas con respecto a la relación entre las distintas manifestaciones artísticas y el perfil del profesional en Estudios Socioculturales para presentarlas en las asignaturas de sus años académicos;

12.Promover, por parte de los grupos de estudiantes con afinidades artísticas específicas, las acciones culturales de la facultad dentro y fuera de la universidad con el fin de fortalecer el Festival de Aficionados, por parte de los grupos de estudiantes con afinidades artísticas;

13. Diagnosticar en los estudiantes de toda la carrera aptitudes para el ejercicio de las distintas manifestaciones deportivas;

14.Promover la cultura de la profesión en las comunidades intra y extrauniversitaria a partir del diagnóstico que se aplique a los estudiantes (Día de los Estudios Socioculturales);

15. Diagnosticar en los estudiantes de toda la carrera conocimientos sobre salud humana;

16. Crear y preparar grupos de estudiantes que impartan temas de salud, fundamentalmente sexual y reproductiva;

17. Crear grupos de estudiantes que impartan talleres sobre habilidades sociales, fundamentalmente Trabajo en Equipo;

18. Diagnosticar habilidades comunicativas en la comunidad universitaria;

19. Desarrollar talleres de habilidades comunicativas;

20. Crear grupos de estudiantes para dar a conocer el quehacer universitario a la provincia a través de los medios de difusión masiva del territorio (prensa plana, radial y televisiva);

21. Diagnosticar el conocimiento en la comunidad de profesores y estudiantes sobre el proceso extensionista; 
22. Diagnosticar necesidades, intereses, expectativas y limitaciones de profesores y estudiantes sobre el proceso extensionista;

23. Crear grupos de trabajo (profesores y estudiantes) para el diseño, implementación y evaluación de la dimensión extensionista de los proyectos educativos de las brigadas.

\section{REFERENCIAS}

DELORS, J. et al. La educación encierra un tesoro. Informe a la UNESCO de la Comisión Internacional sobre la educación para el siglo XXI. Santillana: UNESCO, [1996?]. Disponible en: <www.unesco.org>. Consultado día: 12/2/2013.

DÍAZ, L. F.; ALUM, N.; ORDAZ, M. La responsabilidad social universitaria y el trabajo en equipo. Propuesta de acciones extensionistas para los Estudios Socioculturales. Trabajo de Diploma (Carrera Licenciatura en Estudios Socioculturales) - Universidad de Pinar del Río, Pinar del Río, 2013.

GOLEMAN, D. La inteligencia emocional. Por qué es más importante que el cociente intelectual. México: Ediciones B, 1995.

. La práctica de la inteligencia emocional. Barcelona: Kairós, 1998.

GONZÁLEZ, M.; GONZÁLEZ, G. Extensión educativa. Material en soporte digital para el Módulo: Extensión e investigación científica en instituciones educativas del Diplomado Investigación Educativa. Colombia: Pereira, 1999.

MODELO DEL PROFESIONAL. Licenciatura en estudios socioculturales. Cuba: Ministerio de Educación Superior, 2009.

PROGRAMA NACIONAL DE EXTENSIÓN UNIVERSITARIA. Cuba: Ministerio de Educación Superior, 2004.

TÜNNERMAN, C. La Universidad y los desafios del desarrollo y la democracia. Honduras: Millenium, 1993.

UNESCO. Comunicado. Conferencia Mundial sobre la Educación Superior: La nueva dinámica de la educación superior y la investigación para el cambio social y el desarrollo. París: UNESCO, 2009.

. Conferencia Mundial sobre la Educación Superior. La Educación Superior para el siglo XXI: visión y acción. Madrid: UNESCO, 1998.

Texto recebido em 11 de julho de 2013.

Texto aprovado em 30 de julho de 2013. 